\title{
Surgical repair of muscle laceration: biomechanical properties at 6 years follow-up
}

\author{
Francesco Oliva1 \\ Alessio Giai Via ${ }^{1}$ \\ Olga Kirits $i^{2}$ \\ Calogero Foti ${ }^{3}$ \\ Nicola Maffulli ${ }^{4,5}$
}

1 Department of Orthopaedic and Traumatology, University of Rome "Tor Vergata", School of Medicine, Italy

2 Department of Radiology, Mid Staffordshire NHS Foundation Trust, Stafford UK

${ }^{3}$ Department of Physical and Rehabilitation medicine, University of Rome "Tor Vergata", School of Medicine, Italy

${ }^{4}$ Head of Department of Physical and Rehabilitation Medicine, University of Salerno, Italy

5 Queen Mary University of London, Barts and the London School of Medicine and Dentistry, Centre for Sports and Exercise Medicine, Mile End Hospital, London, UK

\author{
Corresponding author: \\ Francesco Oliva \\ Department of Trauma and Ortopaedic Surgery \\ University of Rome "Tor Vergata" \\ School of Medicine \\ Viale Oxford, 81 \\ 00133 Rome, Italy \\ E-mail: olivafrancesco@hotmail.com
}

\section{Summary}

Muscle injuries are challenging problems for surgeons. Muscle trauma is commonly treated conservatively with excellent outcome results while surgical repair is advocated for larger tears/lacerations, where the optimal goal is restore of function. Repair of muscle belly lacerations is technically demanding because the sutures pull out and the likelihood of clinical failure is high. Different suture techniques have been described but still the best suture is debated. We show a case of a pure vastus medialis muscle laceration surgically repaired at 6 years of follow-up.

KEY WORDS: muscle belly lesion, skeletal muscle laceration, muscle repair, epimysium, fibrous scar.

\section{Introduction}

Direct muscle trauma is a common and disabling problem that is difficult to treat. Most of muscle injuries can be treated non-surgically successfully while severe muscle injury need surgical repair. Traumatic wounds represent approximately $8 \%$ of emergency department presentations and among them muscle lacerations are rare but dramatic injury ${ }^{1}$. Repair of muscle belly lacerations is technically demanding, and the likelihood of clinical failure is high².

The best surgical procedure is yet to be defined and its therapeutic management is still unclear with no definite guidelines ${ }^{3}$. If the injury involves the tendon, it can be repaired with several suture techniques that offer high pullout resistance. Injury that involves the muscle belly or musculotendinous junction is a challenging lesion for surgeons because of the lack of reliable suture methods ${ }^{4}$.

Different suturing methods have been proposed for the management of muscle lacerations. They can be divided into conventional suture technique, such as Kessler stitches, horizontal mattress and figure eight stitches, and complex suture techniques ${ }^{4}$. Modified Kessler suture, modified Mason-Allen suture, combination stitch and muscle suture with augmentation belong to the second group. However still the best suturing technique is yet not known ${ }^{4}$. Optimal suturing of muscles may permit early rehabilitation with a low risk of stitch pullout or re-rupture ${ }^{5}$.

The regenerative capacity of the injured skeletal muscle is limited. Fibrotic tissue forms at the injury site, thus delaying the muscle's functional recovery and predisposing to recurrence ${ }^{6}$. The limited ability of the skeletal muscle to self-regenerate may justify the need of biological or synthetic augmentations for repairing large damage ${ }^{2,7}$. To our knowledge, despite the attempt of researches to apply regenerative medicine in cases of muscle injuries, no data from tests on human subjects are recorded in the literature. Suturing the transected muscle may help the muscle healing, but it does not prevent the formation of scar tissue, thus leading to incomplete muscle functional recovery ${ }^{6}$.

Our scope was to present the case of a 30 years male with complete open traumatic transection of his right distal vastus medialis muscle, the outcome of surgical repair and its functional modification at 6 years follow-up.

\section{Case report}

A Caucasian amateur soccer player 30-year-old male presented to the emergency department with a deep traumatic laceration of the right distal thigh on the me- 
dial side caused by a circular saw. After clinical examination and inspection a complete transverse transection of the distal right vastus medialis was diagnosed (Fig. 1). The laceration of the muscle was repaired surgically. We used simple stitches to suture the muscle; epimysium was also included into the suture (Fig. 2). A single dose of 2 grams e.v. of Cefamandole (Cefam, Magis Farmaceutici S.p.A., Italy ) was administered. After the surgical intervention, a compressive stocking was applied. Regarding the rehabilitation strategy, the patient was advised on self-care techniques which included rest, ice and elevation of the surgically treated lower limb for one week, and the use of pain killers (Paracetamol) whenever he needed to ease the pain. In addition the patient started to perform isometric exercises the next day of the repair and eccentric exercises after 6 weeks and was allowed to full weight bearing as tolerated (but with no flexion at the knee joint for one week) and passive assisted knee flexion one week following the repair. Finally the skin stitches were removed at two weeks.

At the end of the rehabilitation the patient experienced no pain, he had full active and passive range of motion at the hip and knee joints and no muscle weakness was detected. The patient returned to his usual daily activities 3 months post intervention.

The patient was reexamined by one of the authors 6

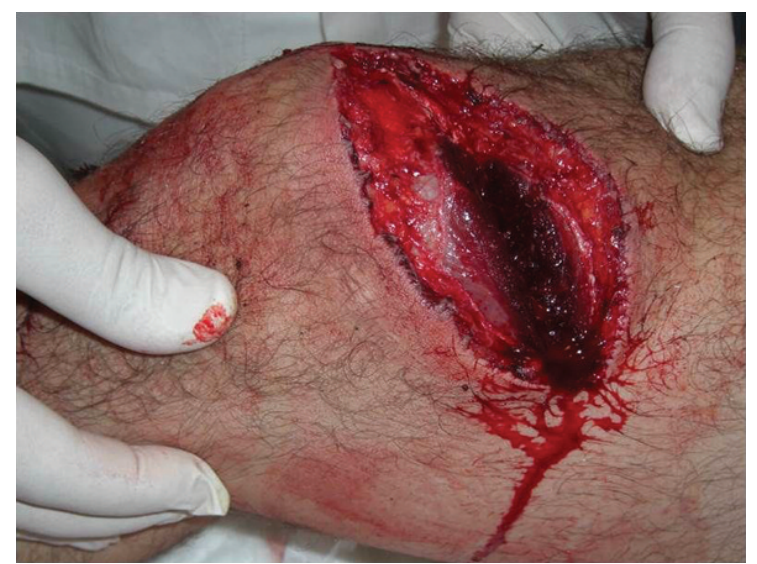

Figure 1. Complete transection of the right vastus medialis muscle. years after the surgical intervention. Patients was satisfied by the surgical outcome and he "rated" his recovery as $100 \%$. He has been pain free with all daily and working activities. He reported no muscle stiffness, numbness or tingling and no re-ruptures or failed repair or new injury was noted. No residual asymmetry was observed during the examination. Adequate tension and continuity were also depicted. Patient reported no functional joint instability during the tests in both legs and knees. Our subject underwent also some functional tests (dynamometric evaluation, isoinertial electromyographic evaluation, linear encoding, $\mathrm{KACLiR}$ test $^{8}$, leg extension test, Half squat position on a vibration platform) (Fig. 3). Cybex dynamometer Isoinertial muscle testing revealed a muscle strength of $86 \%$ compared with the uninjured lower limb. In addition, speed increase of $13 \%$ in the extension of the injured limb compared to the contralateral healthy was noted as well as low activation of the extensor muscles of the injured limb (94\%) compared to the contralateral uninjured limb (132\%). The patient was evaluated also at the same period by MRI. The Magnetic resonance imaging revealed the presence of a scar tissue at the distal vastus medialis muscle, with focal increase of the distal muscle signal intensity probably due to limited fatty atrophy adjacent to the distal surface of the scar (Fig. 4). When compared to the contralateral side no significant interval changes in the bulk of the right vastus medialis belly were recorded.

\section{Discussion}

In our patient who presented with transverse laceration of the distal vastus medialis muscle we used simple stitches to repair the injury with the epimysium sutured to the muscle. The distal location of the laceration was in favor of a better outcome, since the myorrhaphy is easier in the proximal or distal third of the muscle ${ }^{9}$. Also the short time interval between the injury and the surgical intervention was probably a positive predictive factor of muscle recovery. Regarding the type of the used sutures, we know from the literature that the complex stitches have proven superior by showing higher pullout forces than the conventional stitches. However the majority of the articles concern in vitro

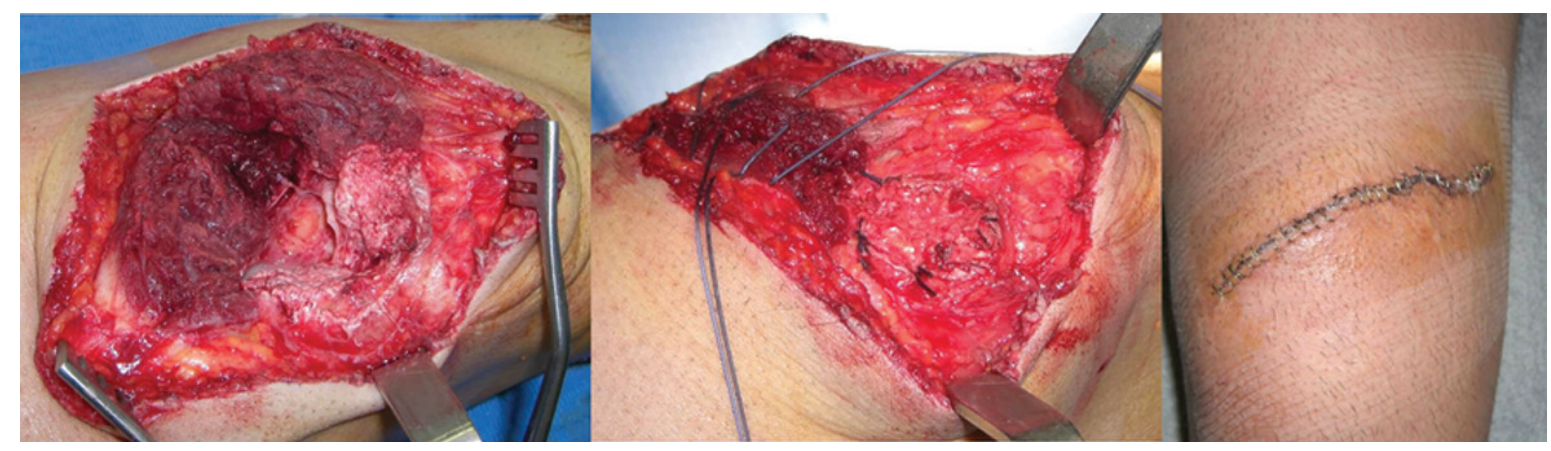

Figure 2. Surgical repair of the muscle laceration, using simple stiches with epimysium. 


\begin{tabular}{|c|c|c|c|c|}
\hline \multicolumn{5}{|c|}{ Leg extension } \\
\hline & $\begin{array}{l}\text { Healthy } \\
\text { Limb }\end{array}$ & $\begin{array}{c}\text { Injured } \\
\text { Limb }\end{array}$ & Ratio l/s \% & Deficit \% \\
\hline Speed $(\mathrm{m} / \mathrm{s})$ & 3,25 & 3,68 & 1,13 & 0,13 \\
\hline Load $(\mathrm{cm})$ & 72,89 & 62,38 & 0,86 & \\
\hline EMG (mV) & 0,18 & 0,21 & 1,18 & \\
\hline
\end{tabular}

Right
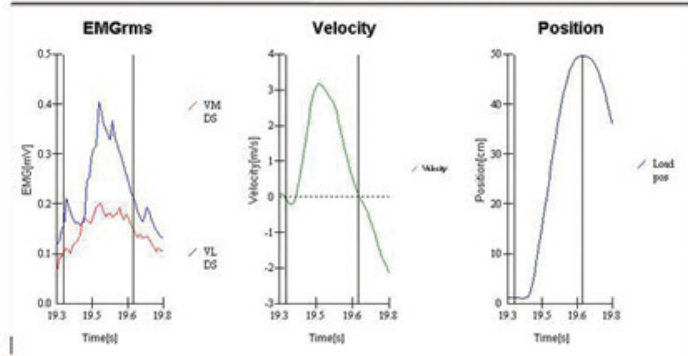

Left
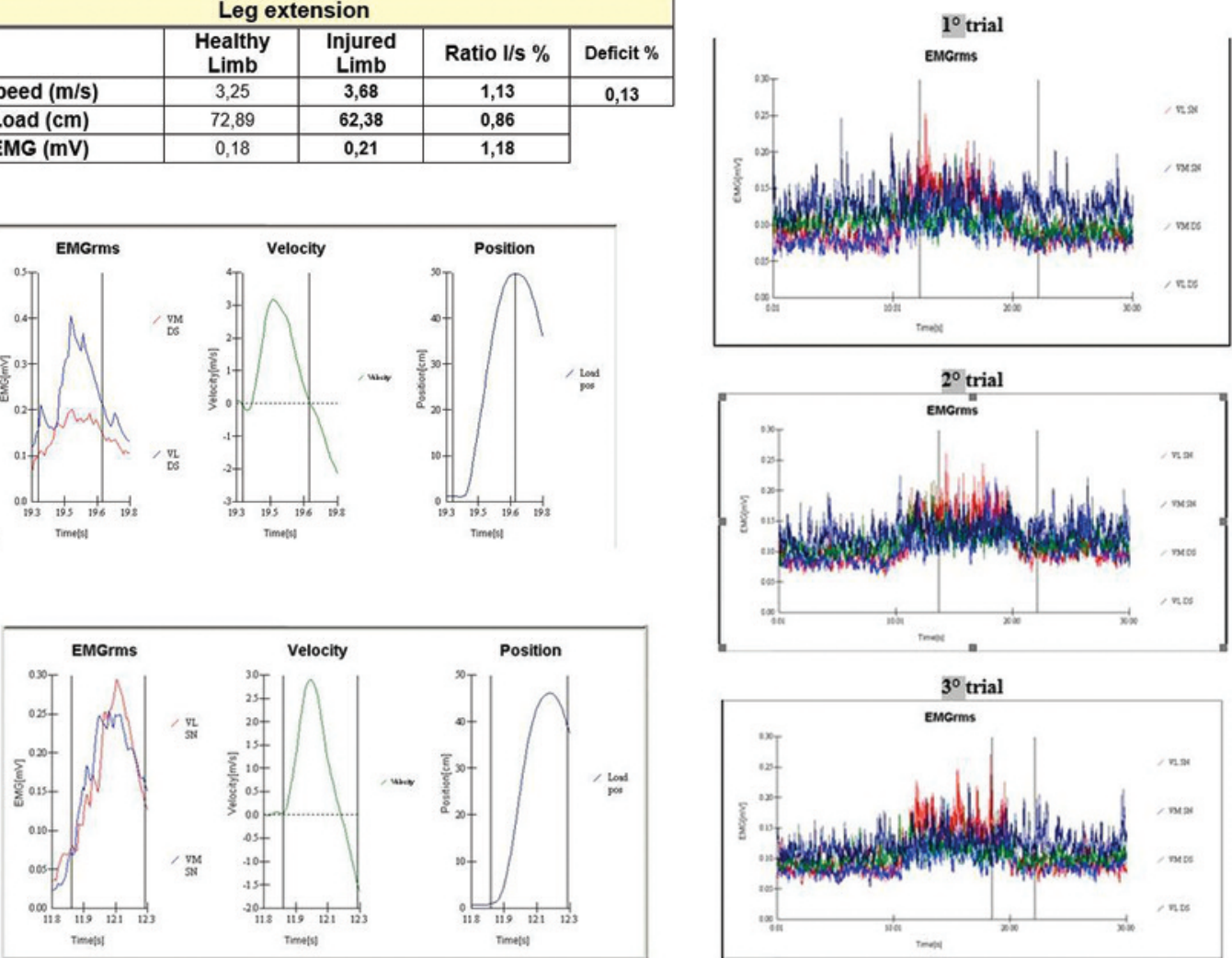

\begin{tabular}{|c|c|c|c|}
\hline \multicolumn{4}{|c|}{ WBV } \\
\hline \hline & HealthyLimb & InjuredLimb & $\begin{array}{c}\text { Deficit } \\
\%\end{array}$ \\
\hline Basal (mV) & 84 & 136 & \\
\hline Vibration (mV) & 111 & 129 & \\
\hline Ratio V/B & $132,00 \%$ & $94,00 \%$ & $\mathbf{- 3 8 , 0 0 \%}$ \\
\hline
\end{tabular}

Figure 3. Functional evaluation of the repaired muscle.

studies or animal studies; in vivo there are no significant differences between the stiches used ${ }^{10}$. In our case, we chose to repair the distal vastus medialis transverse laceration with simple stitches (conventional, non-complex type); as shown by the functional tests our selected suture technique resulted in optimal recovery of the injured muscle. The transverse orientation of the injury could explain the success of the simple stiches used since the latter tend to fail in the longitudinal plane while the complex ones demonstrate failure in the transverse plane ${ }^{11,12}$. The incorporation also of the epimysium significantly improved the biomechanical properties of our sutured muscle belly ${ }^{13}$; the preservation and suturing of the epimysium increased the resistance of the suture to tensile forces ${ }^{13,14}$.

Previous studies have shown that simple stitches could promote fibrosis and lead to exuberant scar tissue by forming a gap deep to the surface ${ }^{11}$. In our patient as shown by the acquired MRI images the biological performance of the simple stiches was optimal and the formed scar tissue was not excessive. This outcome could have been related to the thickness of the vastus medialis muscle at its distal third and thus the decreased depth of the laceration which allowed the simple sutures to pull the tar edges together both at the surface and more deep.

Optimal suturing of muscles may permit early rehabilitation with a low risk of re-rupture or stitch pullout and result in better recovery of the muscles ${ }^{4}$. In our case, the patient was satisfied by the surgical outcome and he "rated" his recovery as $100 \%$. He returned to his pre-injury sporting activities and no re- 


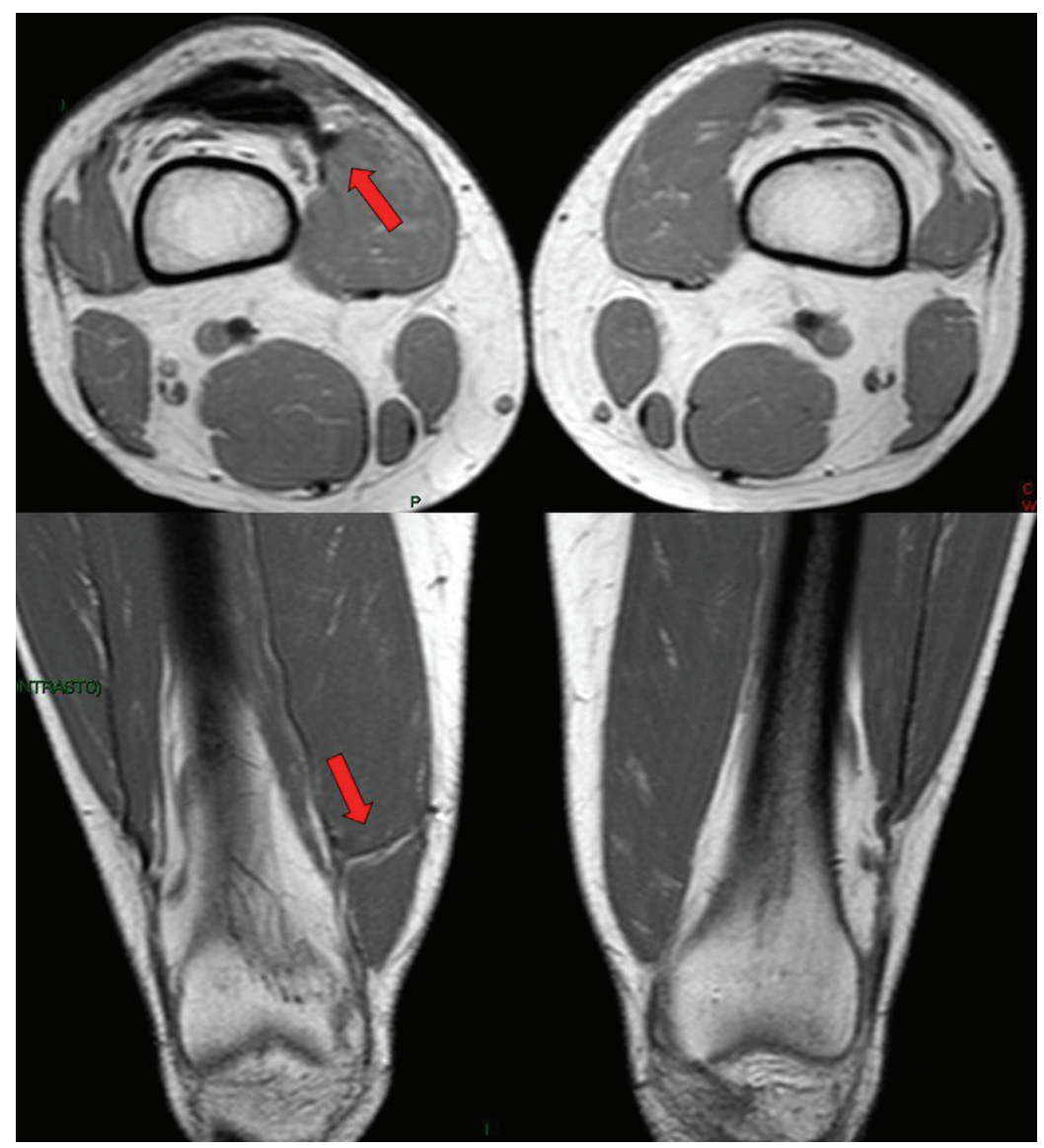

Figure 4. Scar tissue at the distal vastus medialis muscle (red arrow).

ruptures or failed repair or new injury was noted. Return to pre-injury recreational sport activities seemed to be not an issue in our study. Also no asymmetry was observed during the examination, indicative of no frank muscle atrophy especially distally. This clinical assumption was verified at the same time by the appearance of the muscle on the Magnetic resonance imaging; no significant interval change in the Cross Sectional Area (CSA) between the ipsilateral vastus medialis muscle and the healthy contralateral side was observed or significant fatty infiltration of repaired muscle. Regarding the functional evaluation of our patient, Isointertial muscle testing revealed a muscle strength of $86 \%$ compared with the uninjured lower limb, that is $14 \%$ deficit, suggestive of satisfactory recovery of the muscle strength. Regarding the speed of the knee extension, the injured limb achieved better score (13\% speed increase compared to the contralateral healthy). Finally impaired activation of the extensor muscles of the injured limb was observed $(94 \%$, compared to the contralateral uninjured limb, 132\%). It is well known that functional muscular force is the product of both structural (like the muscle size) and neural factors, and compromise to either of these factors impairs the capacity of muscles to exert force. In our patient there was neither significant decrease in the CSA nor fatty infiltration of the injured muscle, but activation impairment of the ipsilateral knee extensors. Therefore we could specu- late that the recorded strength deficit of the injured leg is related maybe partially to the observed lower activation. In addition the increased velocity of the knee extension on the surgically repaired side counteracts the impaired capacity of the injured muscle to produce force, in a subconscious effort of our amateur soccer player during his rehabilitation training to maintain his power; in particular, power is the product of a force on an object and the object's velocity.

In conclusion the muscle belly is not a robust structure and therefore there is no ideal surgical repair method of muscle lacerations, and the outcome of the different suture techniques is related to various biological/biomechanical factors.

\section{References}

1. National Hospital Ambulatory Medical Care Survey. Emergency department summary. National centre for health statistics 1996. Available at: http://www.cdc.gov/nchs/ data/ad/ad293.pdf.

2. Kragh JF Jr, Basamania CJ. Surgical repair of acute traumatic closed transection of the biceps brachii. J Bone Joint Surg Am 2002;84:992-998.

3. Menetrey J, Kasemkijwattana C, Fu FH, Moreland MS, Huard J. Suturing versus immobilization of a muscle laceration. A morphological and functional study in a mouse model. Am J Sports Med 1999;27:222-229.

4. Kragh JF Jr, Svoboda SJ, Wenke JC, Ward JA, Walters TJ. 
Suturing of lacerations of skeletal muscle. J Bone Joint Surg Br 2005;87:1303-1305.

5. Taylor C, Yarlagadda R, Keenan J. Repair of rectus femoris rupture with LARS ligament. BMJ Case Reports 2012. Mar 20.

6. Huard J, Li Y, Fu FH. Muscle injuries and repair: current trends in research. J Bone Joint Surg Am 2002;84:822-832.

7. Rizzi R, Bearzi C, Mauretti A, Bernardini S, Cannata S, Gargioli C. Tissue engineering for skeletal muscle regeneration. Muscles Ligaments Tendons J 2012;2:230-234.

8. Calogero Foti, Laurini A, Tiberti S, et al. Leg extension test, sEMG and vibratory stimuli to assess functional recovery following knee joint surgery. Muscles, ligaments and tendons journal 2012; 2:127-132.

9. Chammout MO, Skinner HB. The clinical anatomy of commonly injured muscle bellies. J Trauma 1986;26:549-552.

10. Chien SH, Chen SK, Lin SY, Chen SS, Wu HS. Repair method and healing of skeletal muscle injury. Gaoxiong Yi Xue Ke Xue Za Zhi. 1991;7:481-488.

11. Chance JR, Kragh JF Jr, Agrawal CM, Basamania CJ. Pullout forces of sutures in muscle lacerations. Orthopedics 2005;28:1187-1190.

12. Cerulli G, Sebastiani E, Placella G, et al. Surgical Treatment. In "Muscle Injuries in Sport Medicine", eds Bisciotti GN and Eirale C, Published on: 2013-09-11.

13. Kragh JF Jr, Svoboda SJ, Wenke JC, Ward JA, Walters TJ Epimysium and perimysium in suturing in skeletal muscle lacerations. J Trauma 2005;59:209-212.

14. Nishimura T, Hattori A, Takahashi K. Ultrastructure of the intramuscular connective tissue in bovine skeletal muscle. A demonstration using the cell-maceration/scanning electron microscope method. Acta Anat (Basel) 1994;151:250257. 